\title{
FLUID OBSERVERS AND TILTING COSMOLOGY
}

\author{
A.A. COLEY, S. HERVIK, W.C. LIM
}

\begin{abstract}
We study perfect fluid cosmological models with a constant equation of state parameter $\gamma$ in which there are two naturally defined time-like congruences, a geometrically defined geodesic congruence and a non-geodesic fluid congruence. We establish an appropriate set of boost formulae relating the physical variables, and consequently the observed quantities, in the two frames. We study expanding spatially homogeneous tilted perfect fluid models, with an emphasis on future evolution with extreme tilt. We show that for ultra-radiative equations of state (i.e., $\gamma>4 / 3$ ), generically the tilt becomes extreme at late times and the fluid observers will reach infinite expansion within a finite proper time and experience a singularity similar to that of the big rip. In addition, we show that for sub-radiative equations of state (i.e., $\gamma<4 / 3$ ), the tilt can become extreme at late times and give rise to an effective quintessential equation of state. To establish the connection with phantom cosmology and quintessence, we calculate the effective equation of state in the models under consideration and we determine the future asymptotic behaviour of the tilting models in the fluid frame variables using the boost formulae. We also discuss spatially inhomogeneous models and tilting spatially homogeneous models with a cosmological constant.
\end{abstract}

\section{INTRODUCTION}

In cosmology it is essential to specify a set of observers, or rather a congruence of world-lines, from which observations are made. Physical quantities, such as the Hubble parameter, depend on the choice of congruence and consequently the Hubble parameter is observer-dependent. In this paper, we will consider a universe with two different congruences of observers, with respect to which the interpretation of the Universe can be radically different. Spatially homogeneous perfect fluid universes in which the fluid is not necessarily comoving with the spatially homogeneous hypersurfaces are prime examples of this phenomenon, and have the advantage that they are dynamical solutions to Einstein's field equations and can model some features of our real Universe. For example, in these models the observers moving with the geometric congruence can experience an ever-expanding non-inflating universe, while observers moving with the matter congruence can experience an inflating universe.

For spatially homogeneous ( $\mathrm{SH}$ ) Bianchi cosmological models, the universe is foliated into space-like hypersurfaces (defined by the group orbits of the respective model) [1, 2, 3, 4. For these perfect fluid models there are two naturally defined time-like vector fields (i.e., congruences): the unit vector field, $n^{a}$, normal to the group orbits and hence orthogonal to the surfaces of transitivity (the 'geometric' congruence), and the four-velocity, $u^{a}$, of the fluid (the 'matter' congruence); see Figure 1. If $u^{a}$ is not aligned with $n^{a}$, the model is called tilted (and non-tilted

Date: October 31, 2018. 


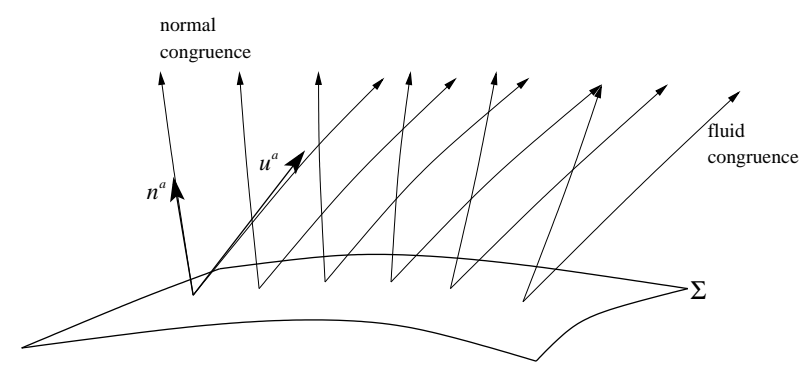

Figure 1. The normal and the fluid congruences defined by the vector fluids $n^{a}$ and $u^{a}$, respectively. The space-like hypersurfaces $\Sigma$ are orthogonal to $n^{a}$ and are defined for each Bianchi types by the group orbits of the respective Lie group.

or orthogonal otherwise) [5]. The geometric congruence is necessarily geodesic, vorticity-free and acceleration-free. The matter congruence, on the other hand, is not necessarily geodesic and can have both vorticity and acceleration. ${ }^{1}$ Usually, the kinematical quantities associated with the normal congruence $n^{a}$ of the spatial symmetry surfaces, rather than the fluid flow $u^{a}$, are used as variables (a comoving fluid description is presented in Appendix A). Following [5], tilt variables $v^{\alpha}$ are introduced, so that in an orthonormal frame where $n^{a}=(1,0,0,0)$, we have

$$
u^{a}=\frac{1}{\sqrt{1-v^{2}}}\left(1, v^{1}, v^{2}, v^{3}\right),
$$

where $v^{2} \equiv v_{\alpha} v^{\alpha}, v^{2} \leq 1$.

We will assume a perfect-fluid matter source with $p=(\gamma-1) \mu$ as equation of state, where $\mu$ is the energy density, $p$ is the pressure, and $\gamma$ is a constant. Causality then requires $\gamma$ to be in the interval $0 \leq \gamma \leq 2$. A positive cosmological constant may also be included in the models. Such SH tilted cosmologies with a $\gamma$-law perfect fluid source have been studied by a number of authors. The tilted Bianchi type II model was studied in [6, the type V model in [7, 8, 9, 10, 11, type $\mathrm{VI}_{0}$ in [12, 13, 14, types IV and $\mathrm{VII}_{h}$ in [15, 16, a subset of the type $\mathrm{VI}_{h}$ models in [15], irrotational type $\mathrm{VII}_{0}$ models in [15, 17], general tilted type $\mathrm{VII}_{0}$ models in [18, and tilted type VIII models in [19.

It is known that the tilt can become extreme asymptotically (i.e., $v^{2} \rightarrow 1$ ). Although it is possible for tilt to be extreme both to the past and to the future, we shall focus on the late-time behaviour of the tilted Bianchi models here. The extreme tilt future attractors in SH models (without a cosmological constant) are summarized in Table 1 (using the notation of [12 - 19]). In particular, let $T$ be the proper time as measured along the fluid congruence. The fluid congruence is usually inconvenient for computation, and so a second congruence utilizing a temporal gauge called the separable volume gauge 20 is used (e.g., the orthogonal frame for $\mathrm{SH}$ models). Let $t$ and $H$ be the proper time and the Hubble scalar associated with this congruence. The separable volume gauge allows a convenient computational time variable $\tau$, called the dynamical time, to be defined globally

\footnotetext{
${ }^{1}$ Perfect fluids with non-zero pressure have non-zero 4 -acceleration when $u^{a} \neq n^{a}$.
} 


\begin{tabular}{|c|c|c|l|}
\hline $\begin{array}{c}\text { Bianchi } \\
\text { model }\end{array}$ & Matter & $\begin{array}{l}\text { Extreme } \\
\text { tilt sink }\end{array}$ & $\begin{array}{l}\text { Other sinks } \\
\text { in set }\end{array}$ \\
\hline \hline $\mathrm{II}$ & $\frac{14}{9}<\gamma<2$ & $\mathcal{E}(\mathrm{II})$ & No \\
\hline $\mathrm{IV}$ & $\frac{6}{5+2 \Sigma_{+}}<\gamma<2,-\frac{1}{4}<\Sigma_{+}<0$ & $\widetilde{\mathcal{L}}_{-}(\mathrm{IV})$ & Yes for $\gamma<\frac{4+\Sigma_{+}}{3}$ \\
& $\frac{3}{2-\Sigma_{+}}<\gamma<2,-\frac{1}{2}<\Sigma_{+}<-\frac{1}{4}$ & $\widetilde{\mathcal{E}}_{-}(\mathrm{IV})$ & No \\
\hline $\mathrm{V}$ & $\frac{6}{5}<\gamma<2$ & $\mathcal{M}_{-}$ & Yes for $\frac{6}{5}<\gamma<\frac{4}{3}$ \\
\hline $\mathrm{VI}_{0}$ & $\frac{6}{5}<\gamma<2$ & $\mathcal{E}\left(\mathrm{VI}_{0}\right)$ & No \\
\hline $\mathrm{VI}_{h}$ & $\tilde{\gamma}_{h}<\gamma<2[*]$ & {$\left[{ }^{*}\right]$} & {$\left[{ }^{*}\right]$} \\
\hline $\mathrm{VII}_{0}$ & $\frac{4}{3}<\gamma<2$ & $\widetilde{P}_{4}\left(\mathrm{VII}_{0}\right)$ & No \\
\hline $\mathrm{VII}_{h}$ & $\frac{6}{5+2 \Sigma_{+}}<\gamma<2,-\frac{1}{4}<\Sigma_{+}<0$ & $\widetilde{\mathcal{L}}_{-}\left(\mathrm{VII}_{h}\right)$ & Yes $(\mathcal{P})$ for $\gamma<\frac{4+\Sigma_{+}}{3}$ \\
& $\frac{3}{2-\Sigma_{+}}<\gamma<2,-\frac{1}{2}<\Sigma_{+}<-\frac{1}{4}$ & $\widetilde{\mathcal{E}}\left(\mathrm{VII}_{h}\right)$ & No \\
\hline $\mathrm{VIII}^{|c|} 1<\gamma<2$ & $\widetilde{E}_{1}(\mathrm{VIII})$ & No \\
\hline
\end{tabular}

TABLE 1. Summary of extremely tilted future attractors. $[*]$ There are extremely tilted attractors in the Bianchi $\mathrm{VI}_{h}$ models, but not all of the sinks have been determined in this case to date; for $-1<h<0, h \neq-1 / 9$, we have that $\tilde{\gamma}_{h}<6 / 5$ (for $h<-1$, we have that $\left.\tilde{\gamma}_{h}<4 / 3\right)$.

for the entire congruence of world-lines:

$$
\frac{\mathrm{d} \tau}{\mathrm{d} t}=H .
$$

For an individual fluid world-line, along which the spatial coordinates are held fixed, we have the following relation between $T$ and $t$ :

$$
\mathrm{d} T=\sqrt{1-v^{2}} \mathrm{~d} t .
$$

We can now express the proper time elapsed, $\Delta T$, along this fluid world-line as an integral over $\tau$ :

$$
\Delta T=T_{2}-T_{1}=\int_{t_{1}}^{t_{2}} \sqrt{1-v^{2}} \mathrm{~d} t=\int_{\tau_{1}}^{\tau_{2}} \frac{1}{H} \sqrt{1-v^{2}} \mathrm{~d} \tau .
$$

Physically, $\tau$ is directly related to the length scale $\ell ; H=\frac{1}{\ell} \frac{\mathrm{d} \ell}{\mathrm{d} t}$ and (2) imply that $\ell=\ell_{0}\left(x^{i}\right) e^{\tau}$. As $\ell \rightarrow \infty$, so does $\tau$. In this paper we are interested in whether $\Delta T$ is finite or infinite as $\tau_{2} \rightarrow \infty$. If $\Delta T$ is finite and $H \rightarrow \infty$ as $\tau_{2} \rightarrow \infty$, then the congruence is future incomplete: the fluid observers will reach infinite expansion within finite proper time. Therefore, in spite of the fact that these spacetimes are future geodesically complete 21, the world-lines defined by the fluid congruences can approach null infinity so quickly that the proper time as measured by the fluid observer is finite.

In this paper, we study perfect fluid cosmological models in which there are two naturally defined congruences, a geometrically defined geodesic congruence and a non-geodesic fluid congruence. In Appendix B we establish an appropriate set of boost formulae relating the physical variables in the two congruences. We study expanding spatially homogeneous tilted perfect fluid models, with an emphasis on future evolution with extreme tilt. In order to set the stage, we shall first discuss the Bianchi type $\mathrm{V}$ model in some detail. We shall then discuss spatially homogeneous 
models with a cosmological constant and various models (Bianchi types $\mathrm{VII}_{0}, \mathrm{VII}_{h}$ and VIII) in the absence of a cosmological constant. We then briefly discuss general spatially inhomogeneous models with a cosmological constant. We find that for $\gamma>$ $4 / 3$, expanding spatially homogeneous tilting universes come to a violent end after a finite proper time, reminiscent to that of cosmological models with a 'phantom energy' equation of state parameter which end in a big rip. We also find that for $\gamma<4 / 3)$ the tilt can become extreme at late times and give rise to an effective quintessential equation of state.

To establish the potential connection with phantom cosmology and quintessence, we determine the effective equation of state in the models under consideration as seen by the fluid observer. In order to investigate the future asymptotic behaviour of the cosmological models we then determine the asymptotic behaviour of the tilting models in the fluid frame variables using the boost formulae derived in Appendix B. We also study the asymptotic dynamical behaviour of a class of LRS Bianchi type $\mathrm{V}$ solutions into the past, and show that they can be extended along the fluid congruence. We conclude with a discussion, with an emphasis on the physical interpretation of the models.

\section{BIANCHI TYPE V}

The irrotational Bianchi type V perfect fluid models were studied in [10]; it was shown that the tilt can become extreme (in a finite time as measured along the fluid congruence). The global structure of Bianchi $\mathrm{V}$ models was studied in more detail in [9]. It was shown that for models with $4 / 3<\gamma \leq 2$, to the future, as $T$ approaches some finite limiting value, the fluid world-lines become null (with respect to the geometric congruence), and the expansion, the shear, the acceleration, and the length scale $\ell$ all diverge but the matter density (and curvature scalars) tend to zero; i.e., the models end at a 'conformal singularity' (this peculiar behaviour is depicted in Figs 7(ii) and 8 in [9]; dynamically an observer is accelerating so quickly that their world-line becomes null near null infinity in finite proper time, signifying the onset of structural instability in the field equations in these models).

This peculiarity occurs for ultra-radiative equations of state. Such an equation of state may not be physically appropriate at late times, although in 22] it was argued that the future is dynamically radiation dominated (but with an always sub-radiative equation of state). This perhaps indicates that models (or rather the governing equations) with an ultra-radiative equation of state may not be wellposed at late times (i.e., structurally unstable). However, we shall show later that a similar type of physical behaviour may occur for particular SH universes with a sub-radiative equation of state.

2.1. Analysis. We shall first show that the fluid proper time is finite as the solution approaches the equilibrium point $M^{-}$for the case $4 / 3<\gamma<2$ in the absence of a cosmological constant (see Fig. 10 in [10]). We shall make use of the evolution equations (denoted by HW) in [10]. There, the equilibrium point $M^{-}$is described by

$$
\Sigma_{+}=\Sigma_{-}=0, \quad A=1, \quad v=-1,
$$

with the density parameter $\Omega=0$ from $\operatorname{HW}(2.13)$ and the deceleration parameter $q=0$ from $\mathrm{HW}(2.14)$. By linearizing equations $\mathrm{HW}(2.10)$ and $\mathrm{HW}(2.11)$ about 
$M^{-}$, we heuristically obtain the decay rates as $\tau \rightarrow \infty$ for the Hubble scalar $H=\theta / 3$ and the quantity $\sqrt{1-v^{2}}$ :

$$
H \approx H_{0} e^{-\tau}, \quad \sqrt{1-v^{2}} \approx\left(\sqrt{1-v^{2}}\right)_{0} \exp \left(-\frac{5 \gamma-6}{2-\gamma} \tau\right),
$$

which have been confirmed by our numerical simulations. Finally, it follows from (4) that the fluid proper time in the future asymptotic regime is

$$
\Delta T \approx \int_{\tau_{0}}^{\infty} \frac{\left(\sqrt{1-v^{2}}\right)_{0}}{H_{0}} \exp \left(-\frac{2(3 \gamma-4)}{2-\gamma} \tau\right) \mathrm{d} \tau,
$$

which is finite for $4 / 3<\gamma<2$, as claimed. This is consistent with the analysis of Collins and Ellis [9] second table in Fig. 7].

2.2. Cosmological constant. Spatially homogeneous cosmological models with a positive cosmological constant were investigated using dynamical systems methods in [23], extending the tilted LRS Bianchi type $\mathrm{V}$ analysis of [10] to the $\Lambda \neq 0$ case. Unlike the case of orthogonal Bianchi models (including type IX), in which the de Sitter point is the only new equilibrium point, when extending these models from the $\Lambda=0$ case tilted models have additional equilibrium points, namely the de Sitter point with extreme tilt for $\gamma \neq 4 / 3$, and with arbitrary tilt for $\gamma=4 / 3$.

In the LRS case, the field equations and conservation equations give rise to a low dimensional constrained dynamical system when written in terms of dimensionless variables. Focusing on the future evolution of these models, all of the sinks were determined. The conventional de Sitter solution is a sink for $\gamma<4 / 3$. The only sinks in the case $\gamma>4 / 3$ are the de Sitter points with extreme tilt $(v= \pm 1)$. The threshold value $\gamma=4 / 3$ is particularly interesting, as a non-zero cosmological constant then implies $v^{\prime}=0$ for any value of $v$. In other words, for each value of the tilt variable $v$, there is a de Sitter equilibrium point [23].

Therefore, in general, for Bianchi type $\mathrm{V}$ models with $\gamma>4 / 3$, the tilt becomes extreme at late times. The fluid motion is no longer orthogonal to the surfaces of homogeneity, and the rest spaces of an observer comoving with the fluid need not be spatially homogeneous and the model will not seem to isotropize. (When following the normal congruence, on the other hand, the fluid will no longer look perfect.)

It has been shown that expanding non-type-IX Bianchi models with a positive cosmological constant isotropize to the future (cosmic no hair theorem) 24]. This result applies to tilted models, but the isotropization is with respect to the congruence normal to the homogeneous symmetry surfaces - not the fluid congruence [25. Thus in the Bianchi type $\mathrm{V}$ models the the space-time generically becomes de Sitter-like, in accordance with the cosmic no hair theorem, but since the tilt does not die away, isotropization of the cosmology does not occur with respect to the fluid congruence. It may be possible to extend beyond the apparent limiting point to a region with time-like symmetries, as discussed in 9 .

\section{Spatially Homogeneous Models}

In general, $\mathrm{SH}$ models are not asymptotically isotropic.

3.1. Isotropic Asymptotia. The spatially homogeneous models isotropize to the future in the presence of a positive cosmological constant [24]. 
3.1.1. Cosmological constant. Let us investigate a $\mathrm{SH}$ model with a cosmological constant and a tilted perfect fluid with $p=(\gamma-1) \mu$. Consider the asymptotic behaviour of the following solution which corresponds to an equilibrium point of the system of governing equations:

Solution: de Sitter (dS) with an extremely tilted perfect fluid.

Asymptotic behaviour [26]: $H=H_{0}, \sqrt{1-v^{2}} \approx\left(\sqrt{1-v^{2}}\right)_{0} \exp \left(-\frac{3 \gamma-4}{2-\gamma} \tau\right)$.

This gives for $\Delta T$ :

$$
\Delta T \approx \int_{\tau_{0}}^{\infty} \frac{\left(\sqrt{1-v^{2}}\right)_{0}}{H_{0}} \exp \left(-\frac{3 \gamma-4}{2-\gamma} \tau\right) \mathrm{d} \tau
$$

which is finite for $4 / 3<\gamma<2$. This solution is the future attractor for $4 / 3<\gamma<2$ and therefore represents the generic behaviour for this model.

3.1.2. Intermediate behaviour. In the absence of a cosmological constant, in general $\mathrm{SH}$ models are not asymptotically isotropic. However, these models can spend a long time close to a Friedmann model corresponding to a saddle point [2]. Therefore, let us consider one non-tilting perfect fluid with $p_{\perp}=\left(\gamma_{\perp}-1\right) \mu_{\perp}$ and one tilting fluid with $p=(\gamma-1) \mu$. We assume that the non-tilting fluid is dominant and has $\gamma_{\perp}<\gamma$. Consider the equilibrium point:

Solution: Friedmann $F$, with $H \approx H_{0} \exp \left(-\frac{3}{2} \gamma_{\perp} \tau\right)$ with extremely tilted second fluid.

Asymptotic behaviour: $\sqrt{1-v^{2}} \approx\left(\sqrt{1-v^{2}}\right)_{0} \exp \left(-\frac{3 \gamma-4}{2-\gamma} \tau\right)$.

This gives for $\Delta T$ :

$$
\Delta T \approx \int_{\tau_{0}}^{\infty} \frac{\left(\sqrt{1-v^{2}}\right)_{0}}{H_{0}} \exp \left(-\frac{3 \gamma-4-\frac{3}{2} \gamma_{\perp}(2-\gamma)}{2-\gamma} \tau\right) \mathrm{d} \tau
$$

In the class of SH models $F$ is a saddle for $2 / 3<\gamma_{\perp}<2$ and represents possible intermediate behaviour. This means that for solutions spending a finite (but arbitrarily long) time close to the saddle $F$, exhibiting quasi-isotropic behaviour consistent with observations [2, the Hubble parameter as measured by the fluid, $H_{\text {fluid }}$, can become arbitrarily large. Of particular interest are the cases when the non-tilting fluid is dust $\left(\gamma_{\perp}=1\right)$ or radiation $\left(\gamma_{\perp}=4 / 3\right)$ for which $\Delta T$ is finite for $\gamma>14 / 9$ and $\gamma>8 / 5$, respectively.

3.2. Anisotropic Asymptotia. There will be different asymptotic end-states depending on Bianchi types (where the criteria may depend on $\gamma$ ). In all of the cases below we have one tilted perfect fluid.

3.2.1. Bianchi type $V I I_{0}$. The investigation of the type $\mathrm{VII}_{0}$ model is important for several reasons. First, the type $\mathrm{VII}_{0}$ model is the most general spatially homogeneous model allowing for the flat Friedmann-Robertson-Walker (FRW) model as a special case. Both the Bianchi type I model and the $\mathrm{VII}_{0}$ generalise the flat FRW model; however, since the Bianchi type I perfect fluid model does not allow for tilt, only the Bianchi type $\mathrm{VII}_{0}$ model can be used to investigate the effect of tilt on the evolution of the universe close to flatness. In addition, the type $\mathrm{VII}_{0}$ model plays an important role in the Bianchi hierarchy. For example, the type $\mathrm{VII}_{0}$ is a special limit of both the 'most general' type VIII and type IX models. Also, unlike the other Bianchi models of solvable type, the type $\mathrm{VII}_{0}$ state space is unbounded. The type $\mathrm{VII}_{0}$ model is not asymptotically self-similar to the future because the 
unbounded growth of one of the curvature variables leads to oscillations in the shear and the curvature variables [2].

The Bianchi type $\mathrm{VII}_{0}$ model with a tilted $\gamma$-law perfect fluid was studied in 18] using expansion-normalised variables and utilizing a formalism adapted to the time-like geodesics orthogonal to the hypersurfaces defined by the type $\mathrm{VII}_{0}$ group action. In particular, the future asymptotic states were determined. Regarding the tilt velocity, it was shown that for fluids with $\gamma<4 / 3$ (which includes the important case of dust, $\gamma=1$ ) the tilt velocity tends to zero at late times, while for a radiation fluid, $\gamma=4 / 3$, the fluid is tilted and its vorticity is dynamically significant at late times. For fluids stiffer than radiation $(\gamma>4 / 3)$, the future asymptotic state is an extremely tilted spacetime with vorticity.

For $4 / 3<\gamma<2$, the solution corresponding to the sink [18] has the asymptotic form

$$
H \approx H_{0} e^{-2 \tau}, \quad \sqrt{1-v^{2}} \approx\left(\sqrt{1-v^{2}}\right)_{0} \exp \left(-\frac{3 \gamma-4}{2-\gamma} \tau\right) .
$$

This implies,

$$
\Delta T \equiv \int_{\tau_{0}}^{\infty} \frac{1}{H} \sqrt{1-v^{2}} \mathrm{~d} \tau \approx \int_{\tau_{0}}^{\infty} \frac{\left(\sqrt{1-v^{2}}\right)_{0}}{H_{0}} \exp \left(-\frac{5 \gamma-8}{2-\gamma} \tau\right) \mathrm{d} \tau
$$

which corresponds to the proper time of an observer from $\tau=\tau_{0}$ to $\tau=\infty$ following the fluid congruence, there is a change of behaviour at $\gamma=8 / 5$. For models with $\gamma \leq 8 / 5$, we find that this integral diverges. However, for fluids with $\gamma>8 / 5$, we find that this integral is finite. Since asymptotically at late times a fluid with $\gamma>$ $8 / 5$ will be extremely tilted, this means that the fluid will reach future null infinity in finite proper time. This is consistent with the work of $[22]^{2}$. As discussed earlier, a similar phenomenon occurs for the LRS Bianchi type V model with $\gamma>4 / 3[9]$.

3.2.2. Bianchi type $V I I_{h}$. For this model the appropriate solutions are vacuum plane waves with extreme tilt (see [15, 16] for details). There are two possible asymptotic states depending on a parameter $\Sigma_{+}$:

(1) $\widetilde{\mathcal{L}}_{-}\left(V I I_{h}\right)$ : Stable for $6 /\left(5+2 \Sigma_{+}\right)<\gamma<2,-1 / 4<\Sigma_{+}<0$. Asymptotic behaviour: $H \approx H_{0} e^{-\left(1-2 \Sigma_{+}\right) \tau}$,

$$
\sqrt{1-v^{2}} \approx\left(\sqrt{1-v^{2}}\right)_{0} \exp \left(-\frac{2\left(5 \gamma-6+2 \gamma \Sigma_{+}\right)}{2-\gamma} \tau\right) .
$$

This gives for $\Delta T$ :

$$
\Delta T \approx \int_{\tau_{0}}^{\infty} \frac{\left(\sqrt{1-v^{2}}\right)_{0}}{H_{0}} \exp \left(-\frac{2\left(3 \gamma-4+2 \Sigma_{+}\right)}{2-\gamma} \tau\right) \mathrm{d} \tau .
$$

(2) $\widetilde{\mathcal{E}}\left(V I I_{h}\right)$ : Stable for $3 /\left(2-\Sigma_{+}\right)<\gamma<2,-1 / 2<\Sigma_{+}<-1 / 4$.

Asymptotic behaviour: $H \approx H_{0} e^{-\left(1-2 \Sigma_{+}\right) \tau}$,

$$
\sqrt{1-v^{2}} \approx\left(\sqrt{1-v^{2}}\right)_{0} \exp \left(-\frac{2\left(1-2 \Sigma_{+}\right)\left(2 \gamma-3-\gamma \Sigma_{+}\right)}{3\left|\Sigma_{+}\right|(2-\gamma)} \tau\right)
$$

\footnotetext{
${ }^{2}$ In particular, the dynamical future of radiation dominated Bianchi $\mathrm{VII}_{0}$ models, regarded as homogeneous perturbations of flat Friedmann model, was studied in 22. At late times it was found that $v \sim \ell^{(3 \gamma-4)}, \omega / H^{2} \sim \ell^{(9 \gamma-10)}$, signifying a growing vorticity instability. It was consequently found that the flat radiation filled Friedmann model is unstable to the development of vorticity.
} 
This gives for $\Delta T$ :

$$
\Delta T \approx \int_{\tau_{0}}^{\infty} \frac{\left(\sqrt{1-v^{2}}\right)_{0}}{H_{0}} \exp \left(-\frac{\left(1-2 \Sigma_{+}\right)^{2}(2 \gamma-3)}{3\left|\Sigma_{+}\right|(2-\gamma)} \tau\right) \mathrm{d} \tau .
$$

In the case $-1 / 2<\Sigma_{+}<-1 / 4$, the asymptotic state is actually a closed orbit, so in calculating the decay rates we have considered the appropriate averaged value as described in Section 4 of [16]. From the above expressions $\Delta T$ is finite whenever $\gamma>\left(4-2 \Sigma_{+}\right) / 3$ for $-1 / 4<\Sigma_{+}<0$ and $\gamma>3 / 2$ for $-1 / 2<\Sigma_{+}<-1 / 4$. However, since for a given type $\mathrm{VII}_{h}$ model any $-1 / 2<\Sigma_{+}<0$ is possible, we have that:

For tilting perfect fluid models of Bianchi type VII $h$ with equation of state parameter $4 / 3<\gamma<2$, there exists a set of solutions of non-zero measure for which $\Delta T$ is finite.

3.2.3. Bianchi type VIII. These models are asymptotically extremely tilted for $1<$ $\gamma<2$ and the asymptotic solution is an extremely Weyl-curvature dominated model (in the language of [27]). The solutions tend to a vacuum state but do so rather slowly: $\mu / H^{2} \propto 1 / \tau$. We refer to 19 for details.

Asymptotic behaviour $(1<\gamma<2): H \approx H_{0} \tau^{\frac{1}{4}} \exp \left(-\frac{3}{2} \tau\right)$,

$$
\sqrt{1-v^{2}} \approx\left(\sqrt{1-v^{2}}\right)_{0} \tau^{\frac{1}{2(2-\gamma)}} \exp \left(-\frac{3(\gamma-1)}{2-\gamma} \tau\right) .
$$

This gives for $\Delta T$ :

$$
\Delta T \approx \int_{\tau_{0}}^{\infty} \frac{\left(\sqrt{1-v^{2}}\right)_{0}}{H_{0}} \tau^{\frac{\gamma}{4(2-\gamma)}} \exp \left(-\frac{3(3 \gamma-4)}{2(2-\gamma)} \tau\right) \mathrm{d} \tau,
$$

which is finite for $4 / 3<\gamma<2$.

\section{General Spatially Inhomogeneous Models with a cosmological CONSTANT}

It is known that for general spatially inhomogeneous perfect fluid models with a cosmological constant, the de Sitter solution with extreme tilt ${ }^{3}$ is locally stable for $4 / 3<\gamma<2$ [26, 21]. The de Sitter asymptotic expansion is given in [26]. From Equations (3.43) and (3.28) of [26], we have that

$$
H \approx H_{0}, \quad \sqrt{1-v^{2}} \approx\left(\sqrt{1-v^{2}}\right)_{0} \exp \left(-\frac{3 \gamma-4}{2-\gamma} \tau\right),
$$

and thus

$$
\Delta T \approx \int_{\tau_{0}}^{\infty} \frac{\left(\sqrt{1-v^{2}}\right)_{0}}{H_{0}} \exp \left(-\frac{3 \gamma-4}{2-\gamma} \tau\right) \mathrm{d} \tau,
$$

which is finite for $4 / 3<\gamma<2$, as required.

A comment on the physical ramification of this result is in order. We note that for $4 / 3<\gamma<2$, the generic behaviour is $v \rightarrow 1$, which implies that inflation does not isotropise an ultra-radiative fluid. Even if inflation is turned off after a certain number of $e$-foldings, $H_{\text {fluid }}$ can become arbitrary large. However, as noted above, this result does not contradict the cosmic no-hair theorem [24], since generically the space-time is dynamically de Sitter-like (in the normal frame).

\footnotetext{
${ }^{3}$ Here the tilt refers to the fluid tilt with respect to a congruence whose acceleration tends to zero.
} 


\section{EfFective Equation of state}

We observe that the future dynamical evolution of the models under investigation (with $\gamma>4 / 3$ ) is reminiscent to that of cosmological models with a constant equation of state parameter $\gamma_{\text {eff }}<0$, dubbed 'phantom energy', in which expanding universes end in a singularity called the big rip after a finite proper time [28]. To establish the connection with physics let us determine the effective equation of state, as measured by the fluid observer, in the models under consideration.

For convenience, we first present the generic boost formula (see Appendix B) for the Hubble scalar:

$$
H_{\text {fluid }}=\Gamma B H \text {, }
$$

where

$$
B=1-\frac{2}{3} v^{\alpha} A_{\alpha}+\frac{1}{3} v^{\alpha} \dot{U}_{\alpha}+\frac{1}{3} \Gamma^{-1} \partial_{0}(\Gamma)+\frac{1}{3} \Gamma^{-1} \partial_{\alpha}\left(\Gamma v^{\alpha}\right) .
$$

A fluid version of (2) can be defined along an individual fluid world-line:

$$
\frac{\mathrm{d} \tau_{\text {fluid }}}{\mathrm{d} T}=H_{\text {fluid }} \text {. }
$$

Then the two dynamical times are related by

$$
\mathrm{d} \tau_{\text {fluid }}=B \mathrm{~d} \tau .
$$

We note that a finite $\Delta T$ implies the divergence of $H_{\text {fluid }}$. Indeed, in the examples above $H_{\text {fluid }}$ diverges.

Let us now consider that the fluid observer interprets the Universe as a flat Friedmann universe with an effective equation of state. We define the effective density $\mu_{\text {eff }}$ and effective pressure $p_{\text {eff }}$ for the fluid frame by writing the Friedmann equation as $0=3 H_{\text {fluid }}^{2}-\mu_{\text {eff }}$, and the Raychaudhuri equation as $\mathbf{e}_{0} H_{\text {fluid }}=$ $-H_{\text {fluid }}^{2}-\frac{1}{6}\left(\mu_{\text {eff }}+3 p_{\text {eff }}\right)$, as is standard. Equivalently, expressed in terms of Hubblenormalized variables, we have $\Omega_{\mathrm{eff}}=1, q_{\mathrm{fluid}}=\frac{1}{2}(\Omega+3 P)_{\mathrm{eff}}$. We define the effective equation of state parameter $\gamma_{\mathrm{eff}}$ as

$$
\gamma_{\mathrm{eff}}=\frac{P_{\mathrm{eff}}}{\Omega_{\mathrm{eff}}}+1
$$

so that $\gamma_{\text {eff }}$ is given by

$$
\gamma_{\text {eff }}=\frac{2}{3}\left(q_{\text {fluid }}+1\right) .
$$

If the limit of $q_{\text {fluid }}$ as $\tau \rightarrow \infty$ exists, then it can be read of from the leading exponent of $H_{\text {fluid }}$ since

$$
H_{\text {fluid }} \approx\left(H_{\text {fluid }}\right)_{0} e^{-\left(q_{\text {fluid }}+1\right) \tau_{\text {fluid }}},
$$

and $\tau_{\text {fluid }}=B \tau$ in the limit. Thus if $H_{\text {fluid }} \approx\left(H_{\text {fluid }}\right)_{0} e^{k \tau}$, then $q_{\text {fluid }}+1=-k / B$ and

$$
\gamma_{\mathrm{eff}}=-\frac{2 k}{3 B}
$$

For the LRS Bianchi type $\mathrm{V}$ models ${ }^{4}$, on the approach to $M^{-}$, we have that

$$
k=\frac{2(3 \gamma-4)}{2-\gamma}, \quad B=\frac{4}{3(2-\gamma)},
$$

\footnotetext{
${ }^{4}$ The effective equation of state in the LRS Bianchi type V models can be calculated directly in fluid-frame variables (see Appendix A.
} 
and so

$$
\gamma_{\mathrm{eff}}=-(3 \gamma-4)<0 \quad \text { for } \gamma>\frac{4}{3}
$$

i.e., on approach to $M^{-}$the ultra-radiative fluid effectively behaves like a phantom energy in an isotropic and spatially flat spacetime ${ }^{5}$.

Similarly, for the de Sitter asymptotic state we have that

$$
k=\frac{3 \gamma-4}{2-\gamma}, \quad B=\frac{2}{3(2-\gamma)},
$$

and coincidentally

$$
\gamma_{\mathrm{eff}}=-(3 \gamma-4)<0 \quad \text { for } \gamma>\frac{4}{3} .
$$

This is the general case in inhomogeneous cosmological models with a cosmological constant. As the de Sitter asymptotic state is approached the ultra-radiative perfect fluid effectively behaves like a phantom energy in an isotropic and spatially flat spacetime. Note that inflation does not stop the big rip from occurring.

Let us now consider the SH models discussed above. For the Bianchi type VIII example, we have that

$$
k=\frac{3(3 \gamma-4)}{2(2-\gamma)}, \quad B=\frac{1}{2-\gamma},
$$

and again

$$
\gamma_{\mathrm{eff}}=-(3 \gamma-4)<0 \quad \text { for } \gamma>\frac{4}{3} .
$$

We also note that for the Bianchi VIII model the future attractor is of extreme tilt for $\gamma>1$, and so $0<\gamma_{\mathrm{eff}}<1$ for $1<\gamma<\frac{4}{3}$.

For the Bianchi type $\mathrm{VII}_{0}$ example, we have

$$
k=\frac{5 \gamma-8}{2-\gamma}, \quad B=\frac{2}{3(2-\gamma)},
$$

and

$$
\gamma_{\mathrm{eff}}=-(5 \gamma-8)<0 \quad \text { for } \gamma>\frac{8}{5} .
$$

We attribute this difference from the previous cases to the fact that Bianchi type $\mathrm{VII}_{0}$ models are geometrically more special than Bianchi type VIII models. For the first Bianchi type $\mathrm{VII}_{h}$ example, we have that

$$
k=\frac{2\left(3 \gamma-4+2 \Sigma_{+}\right)}{2-\gamma}, \quad B=\frac{4\left(1+\Sigma_{+}\right)}{3(2-\gamma)},
$$

and

$$
\gamma_{\mathrm{eff}}=-\frac{3 \gamma-4+2 \Sigma_{+}}{1+\Sigma_{+}}<0 \quad \text { for } \gamma>\frac{4}{3}-\frac{2}{3} \Sigma_{+},
$$

where $\Sigma_{+}$satisfies $-\frac{1}{4}<\Sigma_{+}<0$. And for the second Bianchi type $\mathrm{VII}_{h}$ example, we have that

$$
k=\frac{\left(1-2 \Sigma_{+}\right)^{2}(2 \gamma-3)}{3\left|\Sigma_{+}\right|(2-\gamma)},
$$

\footnotetext{
${ }^{5}$ Note that for $\gamma=\frac{4}{3}$, we obtain an effective equation of state corresponding to a cosmological constant. However, in this case the future attractors are not of extreme tilt and the asymptotic decay rates will differ.
} 
and $B$ is a complicated constant. But since $B$ is always positive, we have that $\gamma_{\text {eff }}<0$ for $\gamma>\frac{3}{2}$. Similarly, for the two-fluid example, we have $\gamma_{\text {eff }}<0$ for $\gamma>\frac{2\left(4+3 \gamma_{\perp}\right)}{3\left(2+\gamma_{\perp}\right)}$. In the generic Bianchi type VIII example the ultra-radiative perfect fluid effectively behaves like a phantom energy. In the more special examples, the requirement is that the equation of state must be equal to or higher than that of radiation.

In order to compare the future asymptotic behaviour with the behaviour of various types of big rip singularities [28, 29, in the next section we shall determine the asymptotic behaviour of the tilting models in the fluid frame variables.

5.1. Quintessence. We also see from above that there are models which have extreme tilt asymptotically, but for $\gamma \leq \frac{4}{3}$ the effective equation of state is that of a cosmological constant $\left(\gamma_{\mathrm{eff}}=0\right)$ or that of quintessence 31 $\left(0<\gamma_{\mathrm{eff}}<\frac{2}{3}\right)$. If $\gamma \leq \frac{4}{3}$, then the future attractor may not be of extreme tilt, in which case the corresponding dynamics is very special. But, from Table 1, there do exist some extreme tilt sinks for $\gamma \leq \frac{4}{3}$. For example, for Bianchi type VIII models there is a unique future attractor $\tilde{E}_{1}$ which is of extreme tilt for all $\gamma>1$, but from equation (28) we see that there are models with $\frac{10}{9}<\gamma<\frac{4}{3}$ that lead to $0<\gamma_{\text {eff }}<\frac{2}{3}$, and thus generically mimic the dynamics of quintessence cosmological models. In the case of Bianchi models of type $\mathrm{V}$, for $\frac{6}{5}<\gamma<\frac{4}{3}$ we again obtain quintessential behaviour (a similar behaviour will occur in type $\mathrm{VI}_{0}$ and $\mathrm{VI}_{h}$ models; however, in all of these cases the extreme tilt sink is not the only future attractor). For certain values of $\Sigma_{+}$(see Table 1 ), the Bianchi type $\mathrm{VII}_{h}$ models also lead to quintessence. We note that spatially homogeneous and inhomogeneous models with a cosmological constant cannot generically give rise to quintessential behaviour since there appear to be no future attractors with extreme tilt when $\gamma<\frac{4}{3}$ (although, of course, the models are already inflating).

\section{Fluid FRAme VARIABLES}

Let us next determine the asymptotic behaviour of the tilting models in the fluid frame variables using the boost formulae given in Appendix B. In particular, we shall present the de Sitter limit and the Bianchi type VIII future asymptotic limits as examples.

For the de Sitter limit, the asymptotic form is given in 26] (eqs. (3.22)-(3.28) and $(3.43))$. Note that the asymptotic form for $v_{\alpha}$ in [26, eqs. (3.27) and (3.28) can be simplified to

$$
v^{\alpha}=v_{0}^{\alpha}+\mathcal{O}\left(e^{-r \tau}\right), \quad 1-v^{2}=e^{-r t}\left[\left(1-v^{2}\right)_{0}+\mathcal{O}\left(e^{-r \tau}\right)\right]
$$

where $r=2(3 \gamma-4) /(2-\gamma)$. That is, the $\mathcal{O}\left(e^{-\tau}\right)$ term is zero. The outline of the proof is as follows; we choose a spatial frame such that $v^{\alpha} \rightarrow(1,0,0)$ and we show that the $\mathcal{O}\left(e^{-\tau}\right)$ term is zero. The result is true for any Fermi-propagated frame. 
With this simplification, we apply the boost formulae in the appendix to the asymptotic forms to obtain the fluid frame variables for the case $\gamma>\frac{4}{3}$ :

$$
\begin{aligned}
H_{\text {fluid }} & =\frac{2}{3(2-\gamma)} \Gamma_{0} \sqrt{\frac{\Lambda}{3}} e^{\frac{1}{2} r \tau}+\mathcal{O}\left(e^{-\frac{1}{2} r \tau}+e^{\left(\frac{1}{2} r-1\right) \tau}\right) \\
a_{\text {fluid }}^{\alpha} & =-\Gamma_{0} \sqrt{\frac{\Lambda}{3}} v_{0}^{\alpha} e^{\frac{1}{2} r \tau}+[\operatorname{coef}] e^{(r-1) \tau}+\mathcal{O}\left(1+e^{\left(\frac{1}{2} r-1\right) \tau}\right) \\
\dot{u}_{\text {fluid }}^{\alpha} & =\frac{2(\gamma-1)}{2-\gamma} \Gamma_{0} \sqrt{\frac{\Lambda}{3}} v_{0}^{\alpha} e^{\frac{1}{2} r \tau}+[\operatorname{coef}] e^{(r-1) \tau}+\mathcal{O}\left(1+e^{\left(\frac{1}{2} r-1\right) \tau}\right) \\
\sigma_{\text {fluid }}^{\alpha \beta} & =\frac{1}{2} r \Gamma_{0} \sqrt{\frac{\Lambda}{3}} v_{0}^{\langle\alpha} v_{0}^{\beta\rangle} e^{\frac{1}{2} r \tau}+[\operatorname{coef}] e^{(r-1) \tau}+\mathcal{O}\left(1+e^{\left(\frac{1}{2} r-1\right) \tau}\right),
\end{aligned}
$$

all of which diverge as $\tau \rightarrow \infty$, while

$$
\omega_{\text {fluid }}^{\alpha \beta}, \Omega_{\text {fluid }}^{\alpha \beta}, n_{\text {fluid }}^{\alpha \beta}=\mathcal{O}\left(1+e^{(r-1) \tau}\right),
$$

which diverge for $\gamma>\frac{10}{7}$.

The factors that contribute to the divergence (in the $e^{\frac{1}{2} r \tau}$ term) are the $\Gamma H$ term (affecting $H_{\text {fluid }}, a_{\text {fluid }}^{\alpha}$ and $\dot{u}_{\text {fluid }}^{\alpha}$ ), and the $\mathbf{e}_{0}$ terms (affecting $H_{\text {fluid }}, \dot{u}_{\text {fluid }}^{\alpha}$ and $\sigma_{\text {fluid }}^{\alpha \beta}$ ). We now look at the general situation for the existence of a singularity. We observe that, in order for a singularity to occur along the fluid congruence, the factor $\Gamma H$ in (44) must diverge sufficiently rapidly. The factor $\Gamma H$ also appears in the boost formulae for $H, a_{\alpha}$ and $\dot{u}_{\alpha}$, so these variables for the fluid frame generally diverge, unless any cancellation occurs. This indicates that a singularity is generally characterized by the divergence of $H, a_{\alpha}$ and $\dot{u}_{\alpha}$. This leads us to speculate that a singularity along a congruence is primarily affected by the rate of expansion and the acceleration of the congruence rather than its shear and vorticity. Furthermore, the spatial curvature component $a_{\alpha}$ plays a more significant role than $n_{\alpha \beta}$ and the frame rotation $\Omega_{\alpha \beta}$ plays a less significant role.

We next present the asymptotic forms for the dominant variables for the Bianchi type VIII limit. From [19, the asymptotic forms for the variables along the congruence normal to the $\mathrm{SH}$ hypersurfaces are

$$
H \approx H_{0} e^{-\frac{3}{2} \tau}, \quad \sigma_{+} \approx \frac{1}{2} H_{0} e^{-\frac{3}{2} \tau}, \quad n_{+} \approx\left(n_{+}\right)_{0} \tau^{-\frac{3}{4}},
$$

$$
\begin{gathered}
1-v^{2} \approx\left(1-v^{2}\right)_{0} \tau^{\frac{1}{2-\gamma}} e^{-\frac{6(\gamma-1)}{2-\gamma} \tau}, \quad v_{1} \approx 1+\mathcal{O}\left(1-v^{2}\right), \quad v_{2}, v_{3} \approx \mathcal{O}\left(\tau^{\frac{3}{4}} e^{-\frac{3}{2} \tau}\right) \\
(42) \quad \sigma_{12}, \sigma_{13} \approx \mathcal{O}\left(\tau^{-\frac{1}{4}} e^{-3 \tau}\right), \quad \sigma_{1} \equiv\left(\sigma_{-}^{2}+\sigma_{\times}^{2}+n_{-}^{2}+n_{\times}^{2}\right) H^{2}=\mathcal{O}\left(\tau^{-1}\right),
\end{gathered}
$$

as $\tau \rightarrow \infty$, valid for $1<\gamma<2$. Applying the boost formulae in the appendix, we obtain

$$
a_{\text {fluid }}^{1} \approx(2-\gamma) H_{\text {fluid }}, \quad \dot{u}_{\text {fluid }}^{1} \approx(2 \gamma-1) H_{\text {fluid }}, \quad\left(\sigma_{+}\right)_{\text {fluid }} \approx(3 \gamma-4) H_{\text {fluid }},
$$

$$
\left(n_{+}\right)_{\text {fluid }} \approx \Gamma_{0}\left(n_{+}\right)_{0} \tau^{-\frac{8-3 \gamma}{4(2-\gamma)}} e^{\frac{3(\gamma-1)}{2-\gamma} \tau},
$$

as $\tau \rightarrow \infty$, while other fluid frame variables are less dominant.

As in the de Sitter example, for $\frac{4}{3}<\gamma<2$, the factor $\Gamma H$ contributes to the divergence of $H_{\text {fluid }}, a_{\text {fluid }}^{1}$ and $\dot{u}_{\text {fluid }}^{1}$, while the $\mathbf{e}_{0}$ terms contribute to the divergence 
of $H_{\text {fluid }}, \dot{u}_{\text {fluid }}^{1}$ and $\left(\sigma_{+}\right)_{\text {fluid }}$. In addition, the diverging factor $\Gamma n_{+}$contributes to the diverging $\left(n_{+}\right)_{\text {fluid }}$, and $\Gamma \sigma_{+}$contributes to $\left(\sigma_{+}\right)_{\text {fluid }}$.

From the asymptotic behaviour of the extremely tilting models in the fluid frame variables we have found that the length scale and the Hubble parameter (i.e., the expansion) diverge in finite proper time, and the singularity is consequently a future sudden singularity in the sense of Barrow 29. In addition, the shear and the acceleration of the fluid congruence also diverge (while the energy density tends to zero). That is, the fluid congruence experiences a 'finite kinematic' singularity, and shares many of the physical properties of a big rip singularity [28]. A 'finite kinematic' singularity is the analogue of a 'conformal' singularity [5] 9] or a 'finite density' singularity [9] (in which the energy density tends to a finite value) that occurs in the Bianchi type $\mathrm{V}$ models. In particular, at a 'finite kinematic' singularity all of the Weyl invariants remain bounded (although components of the Weyl tensor can diverge).

\section{LRS BIANCHI TYPE V EXTENDIBLE SOLUTION}

As noted earlier, a similar situation can occur to the past. As a final example, let us consider the asymptotic dynamical behaviour of a class of Bianchi type $\mathrm{V}$ solutions. Collins and Ellis 9] showed that there exists a set of typical LRS Bianchi $\mathrm{V}$ solutions that, into the past, can be extended along the fluid congruence, from a spatially homogeneous region of spacetime into a stationary region (see their figures 3-7). Along the normal congruence, however, an initial singularity is reached, with the limits

$$
\left(\Sigma_{+}, A, v\right) \rightarrow\left(\Sigma_{+0}, 1+\Sigma_{+0}, 1\right),-1<\Sigma_{+0}<0
$$

and $H \approx H_{0} e^{-\left(1-2 \Sigma_{+0}\right) \tau}, \Gamma \approx \Gamma_{0} e^{-\left(1-2 \Sigma_{+0}\right) \tau}$. The proper time elapsed along the fluid congruence up to the Cauchy horizon is

$$
\Delta T=\int_{-\infty}^{\tau_{0}} \frac{1}{\Gamma H} \mathrm{~d} \tau \approx \int_{-\infty}^{\tau_{0}} \frac{1}{\Gamma_{0} H_{0}} e^{2\left(1-2 \Sigma_{+0}\right) \tau} \mathrm{d} \tau
$$

which is finite.

Here, we apply the boost formulae to the asymptotic forms to check that the fluid frame variables are bounded at the Cauchy horizon, consistent with the extendible 
solution: ${ }^{6}$

$$
H_{\text {fluid }}=-\frac{(2-\gamma) \Sigma_{+0}^{2}+2 \Sigma_{+0}-2 \gamma}{3 \gamma(2-\gamma)} \frac{H_{0}}{\Gamma_{0}}+\mathcal{O}\left(e^{\left(1-2 \Sigma_{+0}\right) \tau}\right)
$$

$$
\left(a_{1}\right)_{\text {fluid }}=\frac{1}{2}\left(1+\Sigma_{+0}\right)\left(1+\Sigma_{+0}-\frac{2}{\gamma} \Sigma_{+0}\right) \frac{H_{0}}{\Gamma_{0}}+\mathcal{O}\left(e^{\left(1-2 \Sigma_{+0}\right) \tau}\right)
$$

$$
\left(\dot{u}_{1}\right)_{\text {fluid }}=-\frac{(\gamma-1)\left[(2-\gamma) \Sigma_{+0}^{2}+2 \Sigma_{+0}-2 \gamma\right]}{\gamma(2-\gamma)} \frac{H_{0}}{\Gamma_{0}}+\mathcal{O}\left(e^{\left(1-2 \Sigma_{+0}\right) \tau}\right)
$$

$$
\left(\sigma_{+}\right)_{\text {fluid }}=\frac{(3 \gamma-4)(2-\gamma) \Sigma_{+0}^{2}-\left(6 \gamma^{2}-18 \gamma+8\right) \Sigma_{+0}+\gamma(3 \gamma-2)}{6 \gamma(2-\gamma)} \frac{H_{0}}{\Gamma_{0}}+\mathcal{O}\left(e^{\left(1-2 \Sigma_{+0}\right) \tau}\right),
$$

as $\tau \rightarrow-\infty$, while other components of the fluid frame variables are identically zero.

Therefore, in this case, although the proper time as measured by an observer is finite, there is a congruence along which the evolution can be continued into the past. This raises the question of whether, for any particular exact cosmological model or corresponding asymptotic state, there exists a congruence whose observers cross a Cauchy horizon into a space-time region not accessible by the observers along the standard congruence of the model considered. In particular, it is of interest to determine whether there is a congruence which can be continued beyond the asymptotic state in any of the examples discussed above in which an extreme tilt future asymptotic state is approached in finite proper time.

\section{Discussion}

We have found that for $\gamma>\frac{4}{3}$ a 'finite kinematic' singularity develops in which the nature of geometrically defined surfaces change from spacelike to null in a finite time as measured by the fluid. The field equations are structurally unstable, and the question consequently arises as of whether these models are mathematically welldefined. In any case, to fully understand the behaviour of these models and their physical properties, the dynamics need to be studied using a formulation adapted to the fluid (i.e., utilizing a fluid-comoving frame).

On the other hand, this mathematical behaviour might lead to some interesting physics. Expanding universes that come to a violent end after a finite proper time have arisen in a different context. Models with a constant equation of state parameter $\gamma<0$, dubbed 'phantom energy', lead to a singularity called the big rip [28. In this paradigm, during the cosmic evolution the length scale grows more rapidly than the Hubble distance and consequently blows up in a finite proper time, and is typically characterized by a divergent pressure and acceleration. As

\footnotetext{
${ }^{6}$ The asymptotic forms for $\Sigma_{+}$and $A$ need to be obtained explicitly up to the order $e^{2\left(1-2 \Sigma_{+0}\right) \tau}$, and $\Gamma$ up to the order $e^{\left(1-2 \Sigma_{+0}\right) \tau}$, as cancellations occur at leading orders to give the eventual bounded limits. Heuristically, we obtain the asymptotic forms as follows: the ansatz $\Sigma_{+}=\Sigma_{+0}+\Sigma_{+1} e^{k_{1} \tau}+\ldots, A=1+\Sigma_{+0}+A_{1} e^{k_{2} \tau}+\ldots$, is substituted into the $\Sigma_{+-}$and $A$ evolution equations and the constraint equations to give $k_{1}=k_{2}=2\left(1-2 \Sigma_{+0}\right)$, and $\Sigma_{+1}$ and $A_{1}$ explicitly in terms of $\Sigma_{+0}$ and $\Gamma_{0}$. This ansatz is then substituted into the $\Gamma$-evolution equation to give $\Gamma=\Gamma_{0} e^{-\left(1-2 \Sigma_{+0}\right) \tau}+\Gamma_{1} e^{\left(1-2 \Sigma_{+0}\right) \tau}$, where $\Gamma_{1}$ is given explicitly in terms of $\Sigma_{+0}$ and $\Gamma_{0}$.
} 
the big rip singularity is approached, both the strong and weak energy conditions are violated. ${ }^{7}$ The details of the pathological behaviours depend on the particular phantom cosmological model under consideration, and less violent singularities such as future sudden singularities are possible [29].

To establish the connection with phantom cosmology we determined the effective equation of state in the models under consideration. We found that as the asymptotic state is approached the ultra-radiative perfect fluid effectively behaves like a phantom energy in an isotropic and spatially flat spacetime. It is important to note that the energy conditions of the perfect fluid are nowhere violated. In order to compare the future asymptotic behaviour with the behaviour of various types of big rip singularities [28] and future sudden singularities [29] we computed the future asymptotic dynamical behaviour of the tilting models.

Let us discuss the physical consequences of this dynamical behaviour in a little more detail. The models presumably spend a period of time approximately isotropic (i.e., close to a flat Friedmann saddle point), with a very small (but non-vanishing) tilt. Thereafter, the models begin to evolve away from isotropy. Since the tilt is non-zero, for $\gamma>\frac{4}{3}$ the models generically evolve towards an asymptotic state with extreme tilt. As the tilt becomes extreme, observers moving with the tilting fluid will experience dynamical behaviour mimicking that of a phantom cosmology. We note that such behaviour seems to be consistent with galaxy, CMB and supernovae observational data [30. Moreover, unlike in conventional phantom cosmology, in the models studied here there is no need for any exotic forms of matter; conventional matter which is tilting suffices. ${ }^{8}$ Indeed, other pathologies, such as the existence of ghosts, are avoided in the models described here. ${ }^{9}$ In addition, as noted above, due to the existence of future attractors with extreme tilt the dynamical behaviour described here is generic.

We have also found that there are tilting SH perfect fluid models with $\gamma<\frac{4}{3}$ which have extreme tilt asymptotically and have an effective quintessence equation of state $\left(0<\gamma_{\mathrm{eff}}<\frac{2}{3}\right)$ 31]. Unlike the case of the cosmologies with $\gamma>\frac{4}{3}$ discussed above in which the phantom behaviour is generic, the quintessential behaviour is typical (i.e., there are other future asymptotic behaviours possible) and model dependent (i.e., it does not occur in all Bianchi models). The details of the extreme tilt sinks in different Bianchi models for $\gamma \leq \frac{4}{3}$ is given in Table 1, and the corresponding ranges of $\gamma$ that lead to $0<\gamma_{\text {eff }}<\frac{2}{3}$ and thus mimic the dynamics of quintessence cosmological models is discussed in Section 5 .

In future work we shall further study the physics of the models with tilt, from the perspective of the observers moving with the fluid matter congruence, using the formalism developed in this paper. We note that the tilting models studied here agree with observations in the same sense that the phantom cosmologies and quintessence models agree with observations, and thus deserve further scrutiny. In particular, we will investigate the effect of tilt on cosmic microwave background

\footnotetext{
${ }^{7}$ We are only considering perfect fluid models here; it may be of interest to investigate more realistic fluids with viscosity.

${ }^{8}$ In a braneworld approach, accelerating universes can also result without a cosmological constant or other form of dark energy [32].

${ }^{9}$ This is also the case in alternative models to phantom cosmology which result from alternative theories of gravity, theories with non-minimal couplings, and models in which the dark energy and quintessence field interact [33].
} 
radiation observations. It is possible that these tilting models may offer an explanation for the low quadrupole amplitude and other anomalies on large angular scales 34 found in the WMAP data [35.

\section{ACKNOWLEDGMENTS}

We thank Henk van Elst for helpful discussions on the boost formulae. This work was supported by a Killam Postdoctoral Fellowship (SH) and the Natural Sciences and Engineering Research Council of Canada (AC).

\section{ApPendix A. LRS BIANCHI TYPE V MODELS IN FLUID-FRAME VARIABLES}

In the LRS Bianchi type $\mathrm{V}$ case, the evolution equations in the fluid frame can be presented as a system of two ODEs, simplified from the equations for general spatially inhomogeneous models ${ }^{10}$ by setting

$$
\begin{gathered}
\boldsymbol{\partial}_{1}=v \boldsymbol{\partial}_{0}, \quad \boldsymbol{\partial}_{2}=\boldsymbol{\partial}_{3}=0, \quad \Sigma_{\alpha \beta}=\operatorname{diag}\left(-2 \Sigma_{+}, \Sigma_{+}, \Sigma_{+}\right), \\
N_{\alpha \beta}=0, \quad A_{\alpha}=(A, 0,0), \quad r_{\alpha}=(r, 0,0), \quad \dot{U}_{\alpha}=(\dot{U}, 0,0), \\
R_{\alpha}=W_{\alpha}=0, \quad P=0, \quad Q_{\alpha}=0, \quad \Pi_{\alpha \beta}=0 .
\end{gathered}
$$

The resulting system is

$$
\begin{aligned}
v^{\prime}= & \left(2 \Sigma_{+}+3 \gamma-4\right) v \\
\Sigma_{+}^{\prime}=- & 3\left(1+\Sigma_{+}\right) G_{-}^{-1}\left[G_{+} \Sigma_{+}+2(\gamma-1)^{2} v^{2}\right] \\
& \quad-\frac{A}{3 v G_{-}}\left[\left(9+v^{2}\right) \Sigma_{+}^{2}+2(6 \gamma-5) v^{2} \Sigma_{+}+(3 \gamma-2) v^{2}\right],
\end{aligned}
$$

where

$$
\begin{aligned}
A & =\frac{-b-\sqrt{b^{2}-4 a c}}{2 a} \\
a & =-\frac{1}{2}(3 \gamma-2) \\
b & =\left[-3 G_{-}+\left(2+G_{-}\right)\left(1+\Sigma_{+}\right)\right] / v \\
c & =\frac{3}{2}(2-\gamma)\left(1+\Sigma_{+}\right)^{2}+3(\gamma-1) G_{-}\left(1+\Sigma_{+}\right) \\
G_{ \pm} & =1 \pm(\gamma-1) v^{2} .
\end{aligned}
$$

The other fluid-frame variables are given by

(62) $\dot{U}=3(\gamma-1) v$

(63) $\frac{r}{v}=q+1=-\frac{3}{G_{-}}\left[G_{+} \Sigma_{+}+2(\gamma-1)^{2} v^{2}\right]-\frac{A}{3 v G_{-}}\left[\left(9+v^{2}\right) \Sigma_{+}+(3 \gamma-2) v^{2}\right]$

(64) $K=2(\gamma-1)\left(1+\Sigma_{+}\right) v^{2}+A^{2}+\frac{2}{3} v\left(1+\Sigma_{+}\right) A$

(65) $\Omega=1-\Sigma_{+}^{2}-K$.

On approach to the $M^{-}$point, $v$ tends to -1 and the fluid-frame variables are:

$$
\Sigma_{+}=-\frac{1}{2}(3 \gamma-4), \quad A=\frac{3}{2}(2-\gamma), \quad \dot{U}=-3(\gamma-1), \quad r=\frac{3}{2}(3 \gamma-4),
$$

which lead to

(67) $\Omega=P=0, \quad \Sigma^{2}=\frac{1}{4}(3 \gamma-4)^{2}, \quad K=\frac{3}{4}(3 \gamma-2)(2-\gamma), \quad q=-\frac{1}{2}(9 \gamma-10)$.

\footnotetext{
${ }^{10}$ See 36 for the general equations in component form.
} 
Many of the calculations are more straightforward in fluid frame variables. For example, using these equations we can easily calculate the asymptotic decay rates (10) and (11) on the approach to $M^{-}$. We can also calculate the effective equation of state (assuming that the effective solution is spatially flat and isotropic, so that $K_{\text {eff }}=0=\Sigma_{\text {eff }}, \Omega_{\text {eff }}=1$ and $\left.q_{\text {fluid }}=\frac{1}{2}(\Omega+3 P)_{\text {eff }}\right)$ :

$$
\gamma_{\text {eff }}=-(3 \gamma-4)
$$

which is negative for $\gamma>\frac{4}{3}$ on approach to $M^{-}$.

\section{Appendix B. Boost Formulae}

The commutation functions are given by:

$$
\left[\mathbf{e}_{a}, \mathbf{e}_{b}\right]=\gamma^{c}{ }_{a b} \mathbf{e}_{c}
$$

which can be decomposed in the following way:

$$
\begin{aligned}
& \gamma_{0 \alpha}^{0}=\dot{u}_{\alpha} \\
& \gamma^{\beta}{ }_{0 \alpha}=-H \delta_{\alpha}{ }^{\beta}-\sigma_{\alpha}{ }^{\beta}-\omega_{\alpha}{ }^{\beta}-\Omega_{\alpha}{ }^{\beta} \\
& \gamma^{0}{ }_{\alpha \beta}=2 \omega_{\alpha \beta} \\
& \gamma_{\alpha \beta}^{\mu}=\varepsilon_{\alpha \beta \nu} n^{\mu \nu}+a_{\alpha} \delta_{\beta}{ }^{\mu}-a_{\beta} \delta_{\alpha}{ }^{\mu}
\end{aligned}
$$

where $\sigma_{\alpha \beta}$ is symmetric and traceless; $n_{\alpha \beta}$ is symmetric; $\omega_{\alpha \beta}$ and $\Omega_{\alpha \beta}$ are antisymmetric. For convenience, we also give the expressions for the components in terms of the commutation functions (angled brackets denote tracefree symmetrization):

$$
\begin{gathered}
H=-\frac{1}{3} \gamma_{0 \mu}^{\mu}, \quad \sigma_{\alpha \beta}=\gamma_{\langle\beta 0 \alpha\rangle}, \quad \omega_{\alpha \beta}+\Omega_{\alpha \beta}=\gamma_{[\beta 0 \alpha]} \\
n^{\alpha \beta}=\frac{1}{2} \varepsilon^{\mu \nu(\beta} \gamma^{\alpha)}{ }_{\mu \nu}, \quad a_{\alpha}=-\frac{1}{2} \gamma^{\mu}{ }_{\mu \alpha} .
\end{gathered}
$$

The boost formulae for the orthonormal frame vector fields are:

$$
\begin{aligned}
\hat{\mathbf{e}}_{0} & =\Gamma \mathbf{e}_{0}+\Gamma v^{\mu} \mathbf{e}_{\mu} \\
\hat{\mathbf{e}}_{\alpha} & =\Gamma v_{\alpha} \mathbf{e}_{0}+B_{\alpha}{ }^{\mu} \mathbf{e}_{\mu}
\end{aligned}
$$

where

$$
B_{\alpha}{ }^{\mu}=\left[\delta_{\alpha}{ }^{\mu}+\frac{\Gamma^{2}}{\Gamma+1} v_{\alpha} v^{\mu}\right], \quad \Gamma=\frac{1}{\sqrt{1-v^{2}}}, \quad v^{2}=v_{\mu} v^{\mu} .
$$

The corresponding boost formulae for the commutation functions are:

$$
\begin{aligned}
\hat{\gamma}^{0}{ }_{0 \alpha}= & \Gamma\left[\hat{\mathbf{e}}_{0}\left(\Gamma v_{\alpha}\right)-\hat{\mathbf{e}}_{\alpha}(\Gamma)\right]-\Gamma v_{\mu}\left[\hat{\mathbf{e}}_{0}\left(B_{\alpha}{ }^{\mu}\right)-\hat{\mathbf{e}}_{\alpha}\left(\Gamma v^{\mu}\right)\right] \\
& +\left(\Gamma B_{\alpha}{ }^{\mu}-\Gamma^{2} v_{\alpha} v^{\mu}\right)\left(\Gamma \gamma^{0}{ }_{0 \mu}-\Gamma v_{\nu} \gamma^{\nu}{ }_{0 \mu}\right) \\
& +\Gamma v^{\mu} B_{\alpha}{ }^{\nu}\left(\Gamma \gamma^{0}{ }_{\mu \nu}-\Gamma v_{\epsilon} \gamma^{\epsilon}{ }_{\mu \nu}\right) \\
\hat{\gamma}^{\beta}{ }_{0 \alpha}= & -\Gamma v^{\beta}\left[\hat{\mathbf{e}}_{0}\left(\Gamma v_{\alpha}\right)-\hat{\mathbf{e}}_{\alpha}(\Gamma)\right]+B_{\mu}{ }^{\beta}\left[\hat{\mathbf{e}}_{0}\left(B_{\alpha}{ }^{\mu}\right)-\hat{\mathbf{e}}_{\alpha}\left(\Gamma v^{\mu}\right)\right] \\
& +\left(\Gamma B_{\alpha}{ }^{\mu}-\Gamma^{2} v_{\alpha} v^{\mu}\right)\left(-\Gamma v^{\beta} \gamma^{0}{ }_{0 \mu}+B_{\nu}{ }^{\beta} \gamma^{\nu}{ }_{0 \mu}\right) \\
& +\Gamma v^{\mu} B_{\alpha}{ }^{\nu}\left(-\Gamma v^{\beta} \gamma^{0}{ }_{\mu \nu}+B_{\epsilon}{ }^{\beta} \gamma_{\mu \nu}^{\epsilon}\right) \\
\hat{\gamma}^{0}{ }_{\alpha \beta}= & 2 \Gamma \hat{\mathbf{e}}_{[\alpha}\left(\Gamma v_{\beta]}\right)-2 \Gamma v_{\mu} \hat{\mathbf{e}}_{[\alpha}\left(B_{\beta]}{ }^{\mu}\right)
\end{aligned}
$$




$$
\begin{aligned}
& +2 \Gamma v_{[\alpha} B_{\beta]}^{\mu}\left(\Gamma \gamma_{0 \mu}^{0}-\Gamma v_{\nu} \gamma_{0 \mu}^{\nu}\right) \\
& +B_{\alpha}{ }^{\mu} B_{\beta}{ }^{\nu}\left(\Gamma \gamma^{0}{ }_{\mu \nu}-\Gamma v_{\epsilon} \gamma^{\epsilon}{ }_{\mu \nu}\right) \\
\hat{\gamma}_{\alpha \beta}^{\gamma}= & -2 \Gamma v^{\gamma} \hat{\mathbf{e}}_{[\alpha}\left(\Gamma v_{\beta]}\right)+2 B_{\mu}{ }^{\gamma} \hat{\mathbf{e}}_{[\alpha}\left(B_{\beta]}{ }^{\mu}\right) \\
& +2 \Gamma v_{[\alpha} B_{\beta]}^{\mu}\left(-\Gamma v^{\gamma} \gamma_{0 \mu}^{0}+B_{\nu}{ }^{\gamma} \gamma^{\nu}{ }_{0 \mu}\right) \\
& +B_{\alpha}{ }^{\mu} B_{\beta}{ }^{\nu}\left(-\Gamma v^{\gamma} \gamma^{0}{ }_{\mu \nu}+B_{\epsilon}{ }^{\gamma} \gamma^{\epsilon}{ }_{\mu \nu}\right)
\end{aligned}
$$

The corresponding boost formulae for the components of the commutation functions are then:

$$
\begin{aligned}
\hat{H}= & \frac{1}{3}\left[\hat{\mathbf{e}}_{\mu}\left(\Gamma v^{\mu}\right)-\frac{\Gamma}{\Gamma+1} v^{\mu} \hat{\mathbf{e}}_{\mu}(\Gamma)+3 \Gamma H-2 \Gamma a_{\mu} v^{\mu}+\Gamma \dot{u}_{\mu} v^{\mu}\right] \\
\hat{\sigma}_{\alpha \beta}= & \hat{\mathbf{e}}_{\langle\alpha}\left(\Gamma v_{\beta\rangle}\right)-\frac{\Gamma}{\Gamma+1} v_{\langle\beta} \hat{\mathbf{e}}_{\alpha\rangle}(\Gamma) \\
& +\Gamma \sigma_{\alpha \beta}+\frac{\Gamma^{4}}{(\Gamma+1)^{2}}\left[v^{2} v^{\mu} \sigma_{\mu\langle\alpha} v_{\beta\rangle}-\sigma_{\mu \nu} v^{\mu} v^{\nu} v_{\langle\alpha} v_{\beta\rangle}\right] \\
& -\Gamma^{2} v^{\mu}\left(\omega_{\mu\langle\alpha}+\Omega_{\mu\langle\alpha}\right) v_{\beta\rangle}+2 \Gamma^{2} v^{\mu} \omega_{\mu\langle\alpha} v_{\beta\rangle} \\
& +\Gamma v^{\mu} \varepsilon_{\mu \nu\langle\alpha} n_{\beta\rangle}+\frac{\Gamma^{3}}{\Gamma+1} v^{\mu} \varepsilon_{\mu \nu\langle\alpha} v_{\beta\rangle} n^{\nu \epsilon} v_{\epsilon} \\
& +\Gamma^{2} a_{\langle\alpha} v_{\beta\rangle}-\frac{\Gamma^{3}}{\Gamma+1} a_{\mu} v^{\mu} v_{\langle\alpha} v_{\beta\rangle} \\
& +\Gamma^{2} \dot{u}_{\langle\alpha} v_{\beta\rangle}-\frac{\Gamma^{3}}{\Gamma+1} \dot{u}_{\mu} v^{\mu} v_{\langle\alpha} v_{\beta\rangle}
\end{aligned}
$$

$\hat{\omega}_{\alpha \beta}+\hat{\Omega}_{\alpha \beta}=\hat{\mathbf{e}}_{[\alpha}\left(\Gamma v_{\beta]}\right)-\frac{\Gamma}{\Gamma+1} v_{[\beta} \hat{\mathbf{e}}_{\alpha]}(\Gamma)+\frac{2 \Gamma}{\Gamma+1} v_{[\beta} \hat{\mathbf{e}}_{0}\left(\Gamma v_{\alpha]}\right)$

$$
+\Gamma^{2} v^{\mu} \sigma_{\mu[\alpha} v_{\beta}
$$$$
+\Gamma\left(\omega_{\alpha \beta}+\Omega_{\alpha \beta}\right)+\frac{\Gamma^{4} v^{2}}{(\Gamma+1)^{2}} v^{\mu}\left(\omega_{\mu[\alpha}+\Omega_{\mu[\alpha}\right) v_{\beta]}+2 \Gamma^{2} v^{\mu} \omega_{\mu[\alpha} v_{\beta]}
$$

$$
\begin{aligned}
& +\Gamma v^{\mu} \varepsilon_{\mu \nu[\alpha} n_{\beta]}^{\nu}+\frac{\Gamma^{3}}{\Gamma+1} v^{\mu} \varepsilon_{\mu \nu[\alpha} v_{\beta]} n^{\nu \epsilon} v_{\epsilon} \\
& +\Gamma^{2} a_{[\alpha} v_{\beta]}+\Gamma^{2} \dot{u}_{[\alpha} v_{\beta]} \\
\hat{\omega}_{\alpha \beta}= & \hat{\mathbf{e}}_{[\alpha}\left(\Gamma v_{\beta]}\right)-\frac{\Gamma}{\Gamma+1} v_{[\beta} \hat{\mathbf{e}}_{\alpha]}(\Gamma) \\
& -\Gamma^{2} v^{\mu} \sigma_{\mu[\alpha} v_{\beta]} \\
& +\Gamma^{2} v^{\mu}\left(\omega_{\mu[\alpha}+\Omega_{\mu[\alpha}\right) v_{\beta]}+\Gamma \omega_{\alpha \beta}-\frac{2 \Gamma^{3}}{\Gamma+1} v^{\mu} \omega_{\mu[\alpha} v_{\beta]} \\
& -\frac{1}{2} \Gamma \varepsilon_{\alpha \beta \mu} n^{\mu \nu} v_{\nu}-\frac{\Gamma^{3}}{\Gamma+1} v^{\mu} \varepsilon_{\mu \nu[\alpha} v_{\beta]} n^{\nu \epsilon} v_{\epsilon} \\
& -\Gamma^{2} a_{[\alpha} v_{\beta]}-\Gamma^{2} \dot{u}_{[\alpha} v_{\beta]} \\
\hat{n}_{\alpha \beta}= & \frac{\Gamma}{\Gamma+1} \varepsilon^{\mu \nu}{ }_{(\alpha \mid} v_{\nu} \hat{\mathbf{e}}_{\mu}\left(\Gamma v_{\mid \beta)}\right)-\frac{\Gamma}{\Gamma+1} \varepsilon^{\mu \nu}{ }_{(\alpha} v_{\beta)} \hat{\mathbf{e}}_{\mu}\left(\Gamma v_{\nu}\right)
\end{aligned}
$$




$$
\begin{aligned}
& -\Gamma v^{\mu} \varepsilon_{\mu \nu(\alpha} \sigma_{\beta)}^{\nu}-\frac{\Gamma^{3}}{\Gamma+1} v^{\mu} \varepsilon_{\mu \nu(\alpha} v_{\beta)} \sigma^{\nu \epsilon} v_{\epsilon} \\
& \left.-\Gamma v^{\mu} \varepsilon_{\mu \nu(\alpha}\left(\omega_{\beta}\right)^{\nu}+\Omega_{\beta}{ }^{\nu}\right)-\frac{\Gamma^{3}}{\Gamma+1} v^{\mu} \varepsilon_{\mu \nu(\alpha} v_{\beta)}\left(\omega^{\nu \epsilon}+\Omega^{\nu \epsilon}\right) v_{\epsilon} \\
& -\Gamma \varepsilon_{\mu \nu(\alpha} v_{\beta} \omega^{\mu \nu}+\frac{2 \Gamma^{3}}{\Gamma+1} v^{\mu} \varepsilon_{\mu \nu(\alpha} v_{\beta)} \omega^{\nu \epsilon} v_{\epsilon} \\
& +\Gamma n_{\alpha \beta}+\frac{\Gamma^{4}}{(\Gamma+1)^{2}}\left[v^{2} v^{\mu} n_{\mu(\alpha} v_{\beta)}-n_{\mu \nu} v^{\mu} v^{\nu} v_{\alpha} v_{\beta}\right] \\
& -\Gamma^{2} v^{\mu} \varepsilon_{\mu \nu(\alpha} v_{\beta)} a^{\nu}-\Gamma^{2} v^{\mu} \varepsilon_{\mu \nu(\alpha} v_{\beta)} \dot{u}^{\nu} \\
& 2 \hat{a}_{\alpha}=\frac{\Gamma}{\Gamma+1} v^{\mu} \hat{\mathbf{e}}_{\mu}\left(\Gamma v_{\alpha}\right)-\frac{\Gamma}{\Gamma+1} v_{\alpha} \hat{\mathbf{e}}_{\mu}\left(\Gamma v^{\mu}\right) \\
& -2 \Gamma H v_{\alpha}+\Gamma^{2} v^{\mu} \sigma_{\alpha \mu}-\frac{\Gamma^{3}}{\Gamma+1} \sigma_{\mu \nu} v^{\mu} v^{\nu} v_{\alpha} \\
& +\Gamma^{2} v^{\mu}\left(\omega_{\alpha \mu}+\Omega_{\alpha \mu}\right)-2 \Gamma^{2} v^{\mu} \omega_{\alpha \mu} \\
& +\Gamma^{2} v^{\mu} \varepsilon_{\mu \nu \alpha} n^{\nu \epsilon} v_{\epsilon} \\
& +\left(2+\Gamma^{2} v^{2}\right) a_{\alpha}-\frac{\Gamma^{4} v^{2}}{(\Gamma+1)^{2}} a_{\mu} v^{\mu} v_{\alpha} \\
& +\Gamma^{2} v^{2} \dot{u}_{\alpha}-\Gamma^{2} \dot{u}_{\mu} v^{\mu} v_{\alpha} \\
& \hat{\dot{u}}_{\alpha}=\hat{\mathbf{e}}_{0}\left(\Gamma v_{\alpha}\right)-\frac{\Gamma}{\Gamma+1} v_{\alpha} \hat{\mathbf{e}}_{0}(\Gamma) \\
& +\Gamma H v_{\alpha}+\Gamma^{2} v^{\mu} \sigma_{\alpha \mu}-\frac{\Gamma^{3}}{\Gamma+1} \sigma_{\mu \nu} v^{\mu} v^{\nu} v_{\alpha} \\
& +\Gamma^{2} v^{\mu}\left(\omega_{\alpha \mu}+\Omega_{\alpha \mu}\right)-2 \Gamma^{2} v^{\mu} \omega_{\alpha \mu} \\
& +\Gamma^{2} v^{\mu} \varepsilon_{\mu \nu \alpha} n^{\nu \epsilon} v_{\epsilon}+\Gamma^{2} v^{2} a_{\alpha}-\Gamma^{2} a_{\mu} v^{\mu} v_{\alpha} \\
& +\Gamma^{2} \dot{u}_{\alpha}-\frac{\Gamma^{3}}{\Gamma+1} \dot{u}_{\mu} v^{\mu} v_{\alpha}
\end{aligned}
$$

The energy-momentum tensor $T_{a b}$ has a standard decomposition relative to a congruence of world-lines:

$$
T_{00}=\mu, \quad T_{0 \alpha}=-q_{\alpha}, \quad T_{\alpha \beta}=p \delta_{\alpha \beta}+\pi_{\alpha \beta},
$$

where $\mu$ is the total energy density, $q_{\alpha}$ is the energy flux, $p$ is the isotropic pressure and $\pi_{\alpha \beta}$ is the trace-free anisotropic pressure, all relative to the above congruence. These variables are called the source terms.

Boosting the orthonormal frame results in the following boost formulae for the source terms:

$$
\begin{aligned}
\hat{\mu} & =\mu+\Gamma^{2} v^{2}(\mu+p)-2 \Gamma^{2} q_{\mu} v^{\mu}+\Gamma^{2} \pi_{\mu \nu} v^{\mu} v^{\nu} \\
\hat{p} & =p+\frac{1}{3} \Gamma^{2} v^{2}(\mu+p)-\frac{2}{3} \Gamma^{2} q_{\mu} v^{\mu}+\frac{1}{3} \Gamma^{2} \pi_{\mu \nu} v^{\mu} v^{\nu} \\
\hat{q}_{\alpha} & =-\Gamma^{2}(\mu+p) v_{\alpha}+\Gamma q_{\alpha}+\frac{\Gamma^{2}(2 \Gamma+1)}{\Gamma+1} q_{\mu} v^{\mu} v_{\alpha}
\end{aligned}
$$




$$
\begin{aligned}
& -\Gamma v^{\mu} \pi_{\mu \alpha}-\frac{\Gamma^{3}}{\Gamma+1} \pi_{\mu \nu} v^{\mu} v^{\nu} v_{\alpha} \\
\hat{\pi}_{\alpha \beta}= & \Gamma^{2}(\mu+p) v_{\langle\alpha} v_{\beta\rangle}-2 \Gamma v_{\langle\alpha} q_{\beta\rangle}-\frac{2 \Gamma^{3}}{\Gamma+1} q_{\mu} v^{\mu} v_{\langle\alpha} v_{\beta\rangle} \\
& +\pi_{\alpha \beta}+\frac{2 \Gamma^{2}}{\Gamma+1} v^{\mu} \pi_{\mu\langle\alpha} v_{\beta\rangle}+\frac{\Gamma^{4}}{(\Gamma+1)^{2}} \pi_{\mu \nu} v^{\mu} v^{\nu} v_{\langle\alpha} v_{\beta\rangle}
\end{aligned}
$$

\section{REFERENCES}

1. G.F.R. Ellis and M.A.H. MacCallum, Comm. Math. Phys. 12 (1969) 108.

2. Dynamical Systems in Cosmology, eds: J. Wainwright and G.F.R. Ellis, Cambridge University Press (1997).

3. A.A. Coley, Dynamical Systems and Cosmology, Kluwer, Academic Publishers (2003).

4. J.D. Barrow and D.H. Sonoda, Phys. Reports 139 (1986) 1.

5. A.R. King and G.F.R. Ellis, Comm. Math. Phys. 31 (1973) 209; G.F.R. Ellis and A.R. King, Comm. Math. Phys. 38 (1974) 119.

6. C.G. Hewitt, R. Bridson, J. Wainwright, Gen. Rel. Grav. 33 (2001) 65.

7. I.S. Shikin, Sov. Phys. JETP 41 (1976) 794.

8. C.B. Collins, Comm. Math. Phys. 39 (1974) 131.

9. C.B. Collins and G.F.R. Ellis, Phys. Rep. 56 (1979) 65.

10. C.G. Hewitt and J. Wainwright, Phys. Rev. D46 (1992) 4242.

11. D. Harnett, Tilted Bianchi type V cosmologies with vorticity, Master's thesis, University of Waterloo (1996).

12. S. Hervik, Class. Quantum Grav. 21 (2004) 2301.

13. A.A. Coley and S. Hervik, Class. Quant. Grav. 21 (2004) 4193.

14. J.D. Barrow and S. Hervik, Class. Quant. Grav. 20 (2003) 2841.

15. A.A. Coley and S. Hervik, Class. Quant. Grav. 22 (2005) 579.

16. S. Hervik, R.J. van den Hoogen and A.A. Coley, Class. Quant. Grav. 22 (2005) 607.

17. W.C. Lim, R. J. Deeley and J. Wainwright, gr-qc/0601040.

18. S. Hervik, R.J. van den Hoogen, W.C. Lim and A.A. Coley, Class. Quant. Grav. 23 (2006) 845 gr-qc/0509031.

19. S. Hervik and W.C. Lim, to appear in Class. Quant. Grav. (2006) gr-qc/0512070.

20. C. Uggla, H. van Elst, J. Wainwright and G. F. R. Ellis, Phys. Rev. D 68 (2004) 103502.

21. A.D. Rendall, Math. Proc. Camb. Phil. Soc. 118 (1995) 511.

22. J.D. Barrow and F.J. Tipler, Nature 276 (1978) 453.

23. M. Goliath and G. F. R. Ellis, Phys. Rev. D 60 (1999) 023502.

24. R.M. Wald, Phys. Rev. D28 (1983) 2118.

25. A.K. Raychaudhuri and B. Modak, Class. Quant. Grav. 5 (1988) 225.

26. W.C. Lim, H. van Elst, C. Uggla and J. Wainwright, Phys. Rev. D 69 (2004) 103507.

27. J.D. Barrow and S. Hervik, Class. Quant. Grav. 19 (2002) 5173.

28. R.R. Caldwell, Phys. Lett. B 545 (2002) 23; B. McInnes, JHEP 0208 (2002) 029; R.R. Caldwell, M. Kamionkowski, N.N. Weinberg, Phys. Rev. Lett. 91 (2003) 071301; L.P. Chimento and R. Lazkoz, Phys. Rev. Lett. 91 (2003) 211301; A. Coley, S. Hervik and J. Latta, astro-ph/0503169.

29. J.D. Barrow, Class. Quant. Grav. 21 (2004) L79; J.D. Barrow and C. Tsagas, Class. Quant. Grav. 22 (2005) 1563.

30. A.G. Riess et al., Ap. J. 607 (2004) 665; U. Seljak et al., Phys. Rev. D 71 (2005) 103515.

31. P.J.E. Peebles and B. Ratra, Ap. J. 325 (1988) L17; C. Wetterich, Nuc. Phys. B 302 (1988) 668 ; R.R. Caldwell, R. Dave, and P.J. Steinhardt, Phys. Rev. Lett. 80 (1998) 1582.

32. G. Dvali, G. Gabadadze and M. Porrati Phys. Lett. B 485 (2000) 208; V. Sahni and V. Yu Shtanov, JCAP 11 (2003) 014.

33. L. Amendola, Phys. Rev. D 62 (2000) 043511; G. Huey and B.D. Wandelt, astro-ph/0407196; S. Das, P.S. Corasanuti and J. Khoury, astro-ph/0510628.

34. T. R. Jaffe, A. J. Banday, H. K. Eriksen, K. M. Gorski and F. K. Hansen, Astrophys. J. 629 (2005) L1 astro-ph/0503213; T. R. Jaffe, S. Hervik, A. J. Banday and K. M. Gorski, submitted to Astrophys. J. astro-ph/0512433. 
35. A. de Oliveira-Costa, M. Tegmark, M. Zaldarriaga and A. Hamilton, Phys. Rev. D 69 (2004) 063516 astro-ph/0307282.

36. H. van Elst, manuscript www.maths.qmul.ac.uk/ hve/onf compo-h.pdf

Department of Mathematics \& Statistics, Dalhousie University, Halifax, Nova ScoTIA, Canada B3H 3J5

E-mail address: herviks@mathstat.dal.ca, wclim@mathstat.dal.ca, aac@mathstat . dal . ca 